\title{
High speed PIV applied to aerodynamic noise investigation
}

\author{
V. Koschatzky $\cdot$ P. D. Moore $\cdot$ J. Westerweel $\cdot$ \\ F. Scarano · B. J. Boersma
}

Received: 11 December 2009/Revised: 26 April 2010/ Accepted: 2 July 2010/Published online: 20 July 2010

(C) The Author(s) 2010. This article is published with open access at Springerlink.com

\begin{abstract}
In this paper, we study the acoustic emissions of the flow over a rectangular cavity. Especially, we investigate the possibility of estimating the acoustic emission by analysis of PIV data. Such a possibility is appealing, since it would allow to directly relate the flow behavior to the aerodynamic noise production. This will help considerably in understanding the noise production mechanisms and to investigate the possible ways of reducing it. In this study, we consider an open cavity with an aspect ratio between its length and depth of 2 at a Reynolds number of $2.4 \times 10^{4}$ and $3.0 \times 10^{4}$ based on the cavity length. The study is carried out combining high speed two-dimensional PIV, wall pressure measurements and sound measurements. The pressure field is computed from the PIV data. Curle's analogy is applied to obtain the acoustic pressure field. The pressure measurements on the wall of the cavity and the sound measurements are then used to validate the results obtained from PIV and check the range of validity of this approach. This study demonstrated that the technique is able to quantify the acoustic emissions from the cavity and is promising especially for capturing the tonal components on the sound emission.
\end{abstract}

V. Koschatzky $(\bowtie) \cdot$ J. Westerweel · B. J. Boersma Laboratory for Aero and Hydrodynamics, Faculty of Mechanical, Maritime and Materials Engineering, Delft University of Technology, Delft, The Netherlands e-mail: v.koschatzky@tudelft.nl

P. D. Moore · F. Scarano

High Speed Laboratory, Faculty of Aerospace Engineering, Delft University of Technology, Delft, The Netherlands

\section{Introduction}

The generation of noise by flow over a rectangular cavity is an important benchmark problem for aeroacoustics and has been investigated both experimentally and numerically in the last decades (Rowley et al. 2002). Because of the relevance for aeronautic applications most of the research has been done on flows at a high Mach number, where compressibility effects are important. Much less attention has been put in low Mach number cavity flows, typical of different ground and submarine applications. Therefore, this work focuses on cavity flows at low Mach numbers to investigate the possibility of estimating the acoustic emission by analysis of PIV data. Ahuja and Mendoza (1995) have shown how the flow that generates past cavities depends on multiple factors such as the cavity geometry, the free stream velocity and the incoming boundary layer properties. Gharib and Roshko (1987) identified different flows behaviors arising in flows over rectangular open ${ }^{1}$ cavities. They showed that, when the ratio between the cavity length and the boundary layer momentum thickness is larger than a certain value ( $L / \theta>80 \div 100$ depending on other parameters) the shear layer separating at the leading edge of the cavity develops in large scale coherent spanwise vortices. They named this flow regime "shear layer mode". At higher values of $L / \theta(>120)$ the "wake mode" occurs. In the "wake mode" the structures become comparable to the cavity dimensions. The external flow alternatively enters into the cavity reattaching to the bottom and ejects out of it. The "wake mode" has been rarely observed in experiments, while it occurs often in numerical simulations

\footnotetext{
${ }^{1}$ A cavity is called open when the ratio between its length and depth is larger than one.
} 
where three-dimensional perturbations of the incoming flow can be completely avoided and periodic boundary conditions are a common practice in the transverse direction, (Larchevêque et al. 2007; Suponitsky et al. 2005). In the "shear layer mode" the vortical structures periodically impinge on the aft wall of the cavity, producing pressure fluctuations that radiate acoustic waves and generate a self-sustaining oscillation mechanism. This mechanism has a double nature: acoustic and hydrodynamic. Which one will predominate depends on the wavelength of the perturbation and therefore on the flow speed and the characteristic dimension of the cavity, the length. In low-speed flows, the cavity can be considered as acoustically compact: its length is much smaller than the acoustic wavelength. Therefore, the acoustic perturbation is not able to influence the flow behavior in the cavity and the self-sustaining oscillation mechanism is purely hydrodynamic. It consists of a recirculating vortex inside the cavity that impinges on the shear layer enhancing and triggering its destabilization. Rossiter (1964) proposed an empirical formula to predict the frequencies of the cavity resonant modes:

$S t=\frac{f L}{U_{0}}=\frac{m-\gamma}{\frac{1}{k}+M a}$.

Here, $S t$ is the Strouhal number, $f$ is the frequency, $L$ the length of the cavity, $U_{0}$ the free stream velocity, $M a$ the Mach number, $k$ the ratio between the shear layer convection velocity and the free stream velocity, $m$ the mode number and $\gamma$ an empirical constant set to 0.25 . The Rossiter equation gives accurate predictions, but fails at very low Mach numbers $(M a<0.2)$, (Howe 1997). The complexity of the flow and its strong time dependence has made investigations difficult and not completely exhaustive. Numerical simulations are limited by the available computational power. While numerical studies are have been performed, at higher Reynolds numbers (increasing turbulence levels) and for complex geometries (such as cavities), due to resolution requirements, modeling of the constitutive equations becomes necessary leading often to results in disaccord with the experimental experience, showing for example a higher occurrence of the "wake mode" (Colonius et al. 1999; Suponitsky et al. 2005). Also few experiments have managed to obtain satisfactory simultaneous measurements of both spatial and temporal variations in a cavity. They have usually been confined to single-point time-resolved measurements or full-field PIV imaging at low speeds, (Ahuja and Mendoza 1995; Block 1976; Grace et al. 2004; Tam and Block 1978). Some more recent attempts, such as that of Haigermoser et al. (2008) and Haigermoser (2009), have performed time-resolved PIV measurements, in water flows and at low free-stream velocity. A noticeable step forward but at conditions that are not yet representative of significant acoustic noise generation. Recent developments in laser and camera technology allow the possibility to extend PIV to the study of aeroacoustic phenomena in air flows at moderate speed (up to $V=20 \mathrm{~m} / \mathrm{s}, \mathrm{Ma}=0.08$ ). The aim of the present study is to make use of these new possibilities, and to obtain estimates of the acoustic emission from time-resolved PIV data. In order to do that we split the problem in two parts: we first compute the hydrodynamic pressure from the PIV velocity fields using the Navier-Stokes equations. The approach we followed is the one proposed by Liu and Katz (2006) and De Kat et al. (2008) further developed by Moore et al. (2009). We then apply Curle's acoustic analogy, in the form suggested by Larsson et al. (2004), to obtain the acoustic pressure field. However, it is well known that aeroacoustic predictions can be sensitive to both measurement and numerical uncertainties. Therefore we test the robustness of our method by comparing the results for the pressure and for the acoustic pressure obtained from PIV with direct measurements of the pressure on the cavity walls and of the emitted sound, by means of several transducers. This approach is only valid in the case of a compact cavity, where the feedback loop triggering the flow instability, that is the source of sound, can be considered as purely hydrodynamic. Therefore, the flow behavior and the acoustic emission are not coupled.

\section{Acoustic pressure prediction from PIV data}

It is worth emphasizing that by using PIV we do not attempt to resolve acoustic fluctuations directly. Usually researchers are interested in acoustic levels at a certain distance from the source. This means that a relevant acoustic field is commonly much larger than the source field. But imaging large areas reduces the spatial resolution of the measurements. Furthermore, the magnitude of the acoustic pressure fluctuations is orders of magnitude smaller than the hydrodynamic pressure fluctuations and certainly cannot be resolved within the measurement certainty. Therefore, it is necessary to model the problem, linking the acoustic emission to quantities that can be measured reliably. Acoustic analogies have been derived that relate the acoustic pressure to flow quantities that are easily obtained from measurements or computation such as hydrodynamic pressure or vorticity (Curle 1955; Ffowcs and Hawkings 1969; Howe 2004, 2003; Lighthill 1952). Those models are obtained under specific assumptions and must be carefully chosen depending on the specific situation. In the present study we apply Curle's analogy, in the form derived by Larsson et al. (2004), to compute acoustic pressure from the PIV data: 


$$
\begin{aligned}
& p(\mathbf{x}, t)-p_{0} \\
& =\left.\frac{1}{4 \pi} \int_{V}\left(\frac{l_{i} l_{j}}{a_{0}^{2} r} \frac{\partial^{2} T_{i j}}{\partial t^{2}} \frac{3 l_{i} l_{j}-\delta_{i j}}{a_{0} r^{2}} \frac{\partial T_{i j}}{\partial t}+\frac{3 l_{i} l_{j}-\delta_{i j}}{r^{3}} T_{i j}\right)\right|_{\left(t-\frac{r}{a_{0}}\right)} \mathrm{d} V(\mathbf{y}) \\
& \quad+\left.\frac{1}{4 \pi} \int_{S} l_{i} n_{j}\left(\frac{\frac{\partial}{\partial t}\left(p \delta_{i j}-\tau_{i j}\right)}{a_{0} r}+\frac{p \delta_{i j}-\tau_{i j}}{r^{2}}\right)\right|_{\left(t-\frac{r}{a_{0}}\right)} \mathrm{d} S(\mathbf{y}),
\end{aligned}
$$

where

$T_{i j}=\rho_{0} u_{i} u_{j}-\tau_{i j}+\left(p-a_{0}^{2} \rho_{0}\right) \delta_{i j}$.

In Eqs. 2 and 3, $u$ is the velocity, $\tau$ the viscous stresses, $p$ the pressure, $l$ a unit vector pointing from the source to the listener position, $n$ the normal to the wall pointing toward the flow, $a_{0}$ the speed of sound, $\delta_{i j}$ the Kronecker delta, and $\rho_{0}$ the fluid density. All terms in the integrals in Eq. 2 are evaluated at the retarded time, $t-r / a_{0}$, where $r=|\boldsymbol{y}-\boldsymbol{x}|$ is the distance between the source and the listener positions. Curle's analogy is particularly suited for the conditions under which the present experiment is performed: the sources due to vortex-vortex interactions, radiating as quadrupoles, and the ones due to the interaction between the vortex and the solid boundaries, radiating as dipoles, are completely isolated. Curle (1955) showed that the ratio of the quadrupole (the volume integral in Eq. 2) and dipole (the surface integral in Eq. 2) source intensities is proportional to the square of the Mach number: $\frac{I_{Q}}{I_{D}} \propto M a^{2}$. We can therefore ignore the volume integral in Eq. 2 given the low speed regime we are investigating. Larsson et al. (2004) showed how, under the same circumstances, viscous terms can be neglected too. In the limit of low Mach number, Eq. 2 can be simplified to:

$p(\mathbf{x}, t)-p_{0}=\left.\frac{1}{4 \pi} \int_{S} l_{i} n_{j}\left(\frac{\partial p}{\partial t} \delta_{i j}+\frac{p \delta_{i j}}{a_{0} r}\right)\right|_{\left(t-\frac{r}{a_{0}}\right)} \mathrm{d} S(\mathbf{y})$.

Equation 4 relates the acoustic pressure at the listener position, $\mathbf{x}$, to a surface integral where only the hydrodynamic wall pressure and its time derivative appear, evaluated at a retarded time that takes into account the distance between the source and the listener position.

The main issue is then to compute the pressure and its time derivative at the walls from the PIV data. In order to do so, we solve a Poisson equation for the pressure derived from the conservation of momentum equation:

$\nabla p=-\rho_{0}\left(\frac{\partial \mathbf{u}}{\partial t}+\mathbf{u} \cdot \nabla \mathbf{u}\right)+v \nabla^{2} \mathbf{u}$.

We ignore the viscous terms on the right-hand side of Eq. 5 . The motivation that lead us to this choice are explained in Sect. 2.1. To compute the time derivatives of the two velocity components in Eq. 5 we use the method proposed by Liu and Katz (2006) and recently developed by Moore et al. (2009). The method consists of the evaluation of the total derivative of the velocity by tracking a fluid parcel following a Lagrangian approach, under the assumption of Taylor's hypothesis of frozen turbulence. This is permitted since the characteristic fluctuations of the flow are small with respect to the characteristic velocity of the mean recirculating flow in the cavity and the larger structures that are responsible for the major fraction of the total sound emission, convect without much deformation within the duration of our acquisition. This approach is necessary due to the relatively large time steps between PIV snapshots that do not allow a correct computation of time derivatives if done in the conventional Eulerian way. Equation 5 can be finally rewritten as:

$\nabla p=-\rho_{0}\left(\frac{D \mathbf{u}}{D t}\right)$.

By taking the divergence of Eq. 6, we derived the Poisson equation for the pressure:

$\nabla^{2} p=-\rho_{0} \nabla \cdot\left(\frac{D \mathbf{u}}{D t}\right)$.

It is worth to point out how in Eq. 7 the time derivatives of the velocities, integrated in the material derivative, must be kept into account. The conservation of mass, in fact, for planar data where only two components of the velocity are determined, is not fully satisfied. This despite the fact that our flow geometry and mean flow may be considered two-dimensional. Equation 7 is solved using a conventional iterative scheme, in which the spatial derivatives are computed by a central difference scheme on a five-point stencil.

Neumann boundary conditions are applied at the boundaries of the domain, as shown in Fig. 1:

$\frac{d p}{d y_{1}}=-\rho_{0} \frac{D u_{1}}{D t} \quad$ On $y_{1}=$ constant boundaries

and

$\frac{d p}{d y_{2}}=-\rho_{0} \frac{D u_{1}}{D t} \quad$ On $y_{2}=$ constant boundaries.

Where $y_{1}$ and $y_{2}$ are the in-plane coordinate direction and $u_{1}$ and $u_{2}$ the corresponding velocity component. Dirichlet

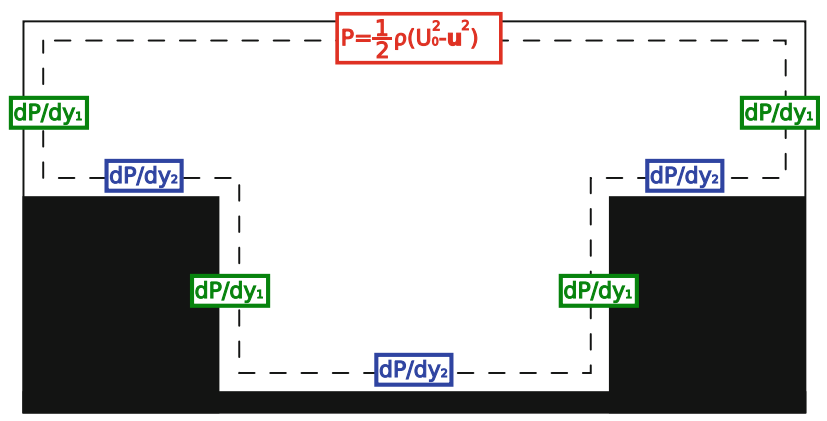

Fig. 1 Boundary condition for the Poisson equation 
boundary conditions are applied at the upper limit of the domain:

$p=\frac{1}{2} \rho_{0}\left(U_{0}^{2}-\mathbf{u}^{2}\right)$.

\subsection{On the viscous terms}

We follow the approach suggested by Liu and Katz (2006). In their paper they point out that in a high Reynolds number flow field located away from boundaries, the material acceleration is dominant and the viscous term is negligible. Additionally their results confirmed that, for cases comparable to our own, the material acceleration is the dominant term in Eq. 5 and the other terms can be neglected. They also note that one should be careful in evaluating the contribution of the viscous term and avoid integrating along paths that are particularly affected by viscosity, e.g., along a boundary layer. With our set of equations we can say the following: ignoring viscous terms in Eq. 5 only affects the Neumann boundary conditions (8) and (9). The Poisson Eq. 7 would be without the viscous terms anyway, because of the mass conservation. Due to the relatively high Reynolds numbers we are considering in this study, the viscous effects are significant only in a small region close to the wall. The computation of the pressure does not need to be carried out fully to the present walls. We then apply the boundary conditions not directly at the walls but 2 vectors rows (corresponding to a distance of $0.96 \mathrm{~mm}$ ) away from them. A distance considered sufficient to be surely out of the viscous layer, but close enough to estimate correctly the pressure at the walls (the pressure does not vary significantly in the direction normal to the wall). Moreover, the computation of those terms involves a double spatial differentiation of the velocity data field, an operation that strongly amplifies signal noise which will lead to a dominating contribution to the final result. Figure 2 shows the mean of the pressure gradient of Eq. 5, the contribution of the acceleration term and the contribution of the viscous term magnified 100 times (Fig. 3). It is clear that the viscous term has high values close to the walls. But its magnitude is around two orders of magnitude lower than the acceleration term. As a result, the total pressure gradient is not substantially affected by ignoring the viscous term. The results in Sect. 5 also confirm the validity of our assumption.

\section{Experimental setup}

The study was carried out combining high-speed planar PIV imaging, wall pressure measurements, and sound measurements. The boundary layer was also measured at the leading edge of the cavity with a hot wire anemometer. We performed experiments with a laminar boundary layer flow

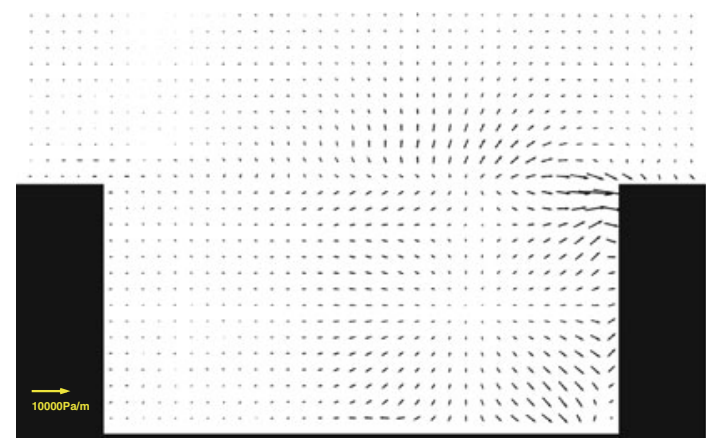

(a) Pressure gradient $\left[\frac{\mathrm{Pa}}{\mathrm{m}}\right]$

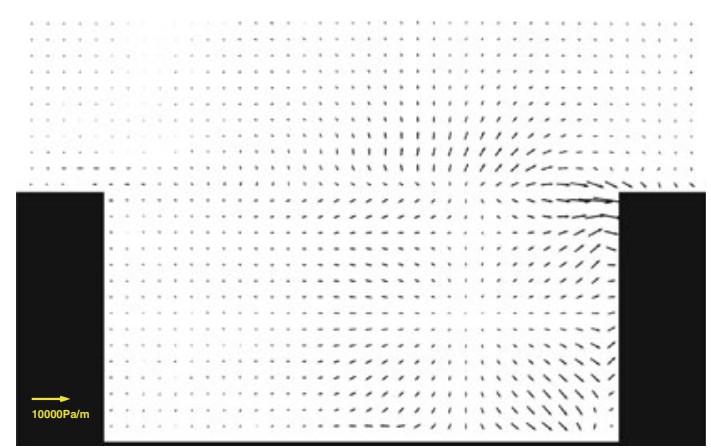

(b) Acceleration term $\left[\frac{\mathrm{Pa}}{\mathrm{m}}\right]$

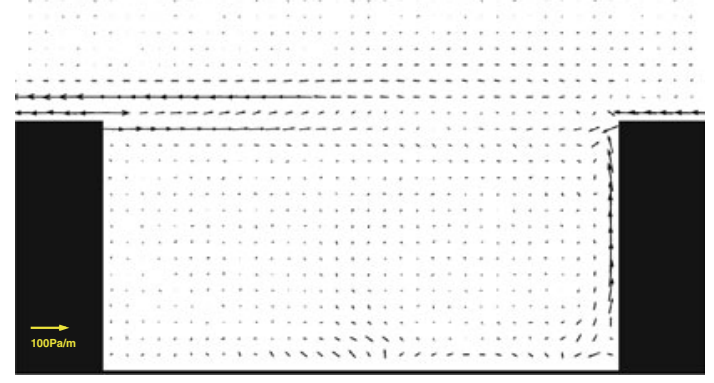

(c) Viscous term, 100 times magnified $\left[\frac{\mathrm{Pa}}{\mathrm{m}}\right]$

Fig. 2 Mean pressure gradient at $12 \mathrm{~m} / \mathrm{s}$. Total, acceleration term and viscous term. One vector every two is displayed

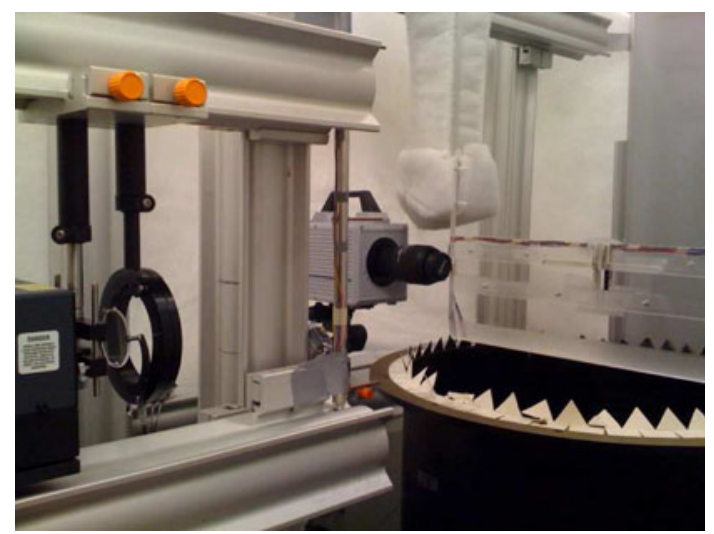

Fig. 3 The experimental setup 
approaching the cavity, at free-stream velocities of 12 and $15 \mathrm{~m} / \mathrm{s}$, giving a Reynolds number of $2.4 \times 10^{4}$ and $3.0 \times 10^{4}$ based on the cavity length, $L$. We choose this Reynolds number range to investigate how the acoustic emission of a cavity in the "shear layer mode" change at different velocities. At lower velocities the approaching boundary layer is too thick compared to the cavity length for the self sustained oscillations to occur. At higher velocities, the incoming boundary layer becomes turbulent and thicker again so the same problem occurs. For the investigated flow condition, the ratio between the length of the cavity and the measured boundary layer momentum thickness is $L / \theta=104$ and 112 respectively, see Table 1 . Measurements were conducted in the vertical open jet wind tunnel of the Low-speed Aerodynamics Laboratories of the Aerospace Department at the Delft University of Technology. The cavity was machined in a flat plate and positioned in the test section aligned with the flow. Figure 4 shows a schematic representation of the experimental configuration including the dimensions of the cavity. The cavity had a width of $600 \mathrm{~mm}$ spanning the full width of the tunnel and giving a width to length ratio of $W / L=20$ (Fig. 5).

\subsection{PIV setup}

The PIV system consisted of a 12-bit 1,024 × 1,024 pixels camera, used at a resolution of $512 \times 1,024$ pixels allowing the required frame rate, (Photron FastCAM SA1) and a dual-cavity pulsed Nd:YLF laser (New Wave Pegasus). The flow was seeded by means of a stage smoke generator (Safex) that produces particles of approximately $1 \mu \mathrm{m}$ in diameter. PIV image pairs were acquired at a frequency of $3 \mathrm{kHz}$ with a time delay between the first and second frame of $80 \mu$ s for the $12 \mathrm{~m} / \mathrm{s}$ free-stream velocity flow and $65 \mu \mathrm{s}$ for the $15 \mathrm{~m} / \mathrm{s}$ one. Those time delays where chosen as an optimum trade-off allowing enough particles displacement between the two frames (in the order of 2-3 pixels inside the cavity) in the slower regions of our flow keeping it within reasonable values in the faster regions (typically 16 pixels in the free-stream area). The laser sheet was estimated by projection onto a millimetric scale and appeared to be approximately $1 \mathrm{~mm}$ thick, a dimension comparable to our final interrogation window size. Data were processed using a multi-pass algorithm, (Westerweel et al. 1997) with the interrogation window sizes tailored to the local flow regime. This approach was chosen to deal with the large dynamic

Table 1 Boundary layer thickness and momentum thickness for the different testing velocities and incoming boundary layers

\begin{tabular}{lll}
\hline$U_{0}(\mathrm{~m} / \mathrm{s})$ & $\delta(\mathrm{mm})$ & $L / \theta$ \\
\hline 12 & 2.30 & 104 \\
15 & 2.14 & 112 \\
\hline
\end{tabular}

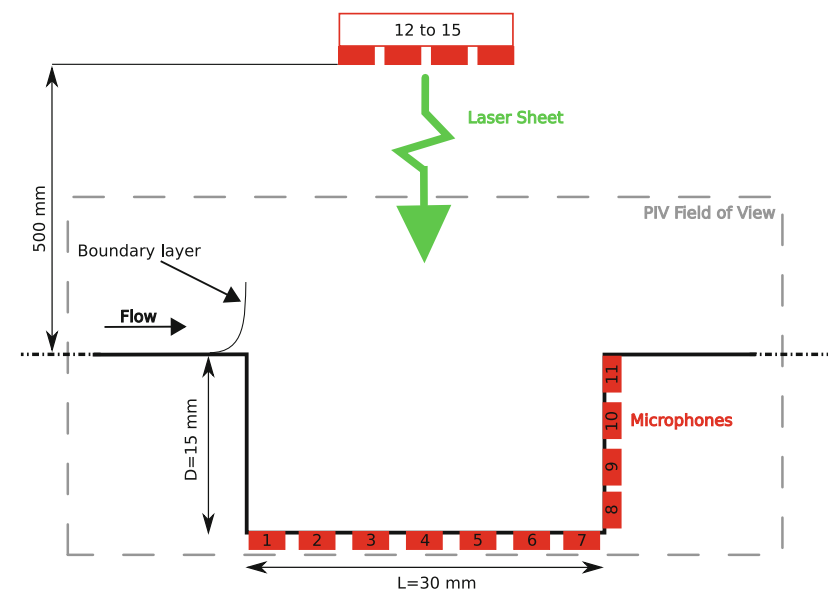

Fig. 4 Schematic representation of the experimental setup. The microphones are marked in red with a reference number

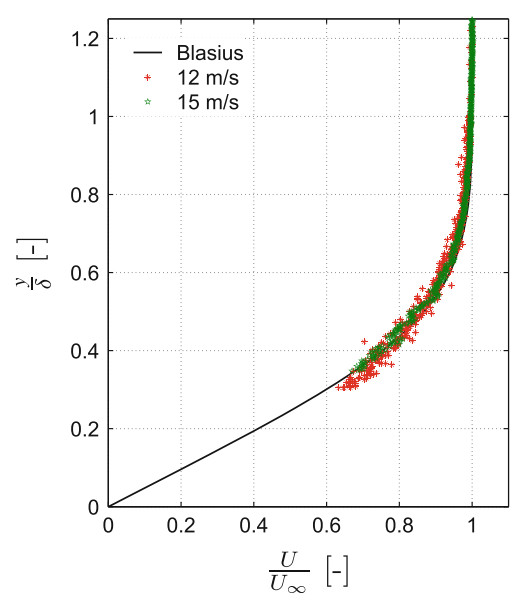

Fig. 5 Boundary layer profiles upstream of the cavity, measured with a hot-wire anemometer

range of the flow. Figure 6 shows the different areas in which the domain was divided and Table 2 gives the initial and intermediate dimensions of the interrogation domains. The incoming boundary layer region was particularly sensitive to the processing parameters because of the strong gradients in the wall-normal direction. The interrogation windows were placed in such a way that their boundaries coincided with the walls of the cavity. The distance between the cavity walls and the first vector is therefore half a window size. The final passes in all domains were done with square interrogation windows of $16 \times 16$ pixels with a $50 \%$ overlap, giving a vector spacing of $0.48 \mathrm{~mm}$.

\subsection{Wall pressure and sound measurements}

The wall pressure fluctuations were measured with 11 microphones (Sonion serie8000), mounted flush with the cavity walls. The microphones were used here as highsensitivity and high-frequency pressure transducers. The 


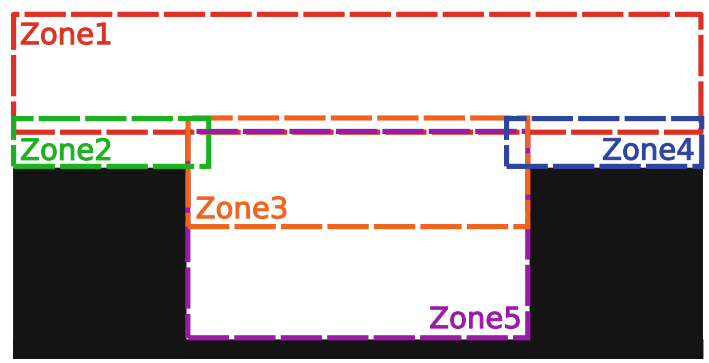

Fig. 6 Various defined domains for the interrogation of the PIV images; see text for explanation

emitted sound was recorded with 4 of the same microphones positioned $0.5 \mathrm{~m}$ above the cavity lid plane. Sound pressure and wall pressure measurements were acquired at a frequency of $100 \mathrm{kHz}$ during the acquisition of the PIV data. The analog microphone signals where amplified, with a separate amplification factor for every microphone in order to get the maximum signal resolution for each microphone. The dynamic range of the measurements was in fact very wide measuring with the same instrument both the wall pressure fluctuations $(\sim 10 \mathrm{~Pa})$ and the acoustic pressure fluctuations $\left(\sim 10^{-1} \mathrm{~Pa}\right)$. The microphones were then calibrated a posteriori by comparison with a calibrated microphone (CESVA P-05) that was exposed together with the other microphones used in the experiment (including the analog signal amplifiers) to the same acoustic field. This field was generated by a signal generator unit and a loudspeaker. We performed measurements for a wide range of amplitudes and frequencies. As specified by the manufacturer of the microphones the amplitude response appeared to be independent to the frequency in our measurement range (from $100 \mathrm{~Hz}$ and up to $8 \mathrm{kHz}$ ). The conversion from electric potential (in Volts) to pressure (in $\mathrm{Pa}$ ) appeared to be given by a linear relation (the coefficient of determination, $R^{2}$, is better than 0.99 ) and with a zero offset, i.e. the conversion reduces to a single gain coefficient.

\section{Flow characterization}

The mean flow is characterized by a large clockwise rotating region in the cavity and a smaller recirculation

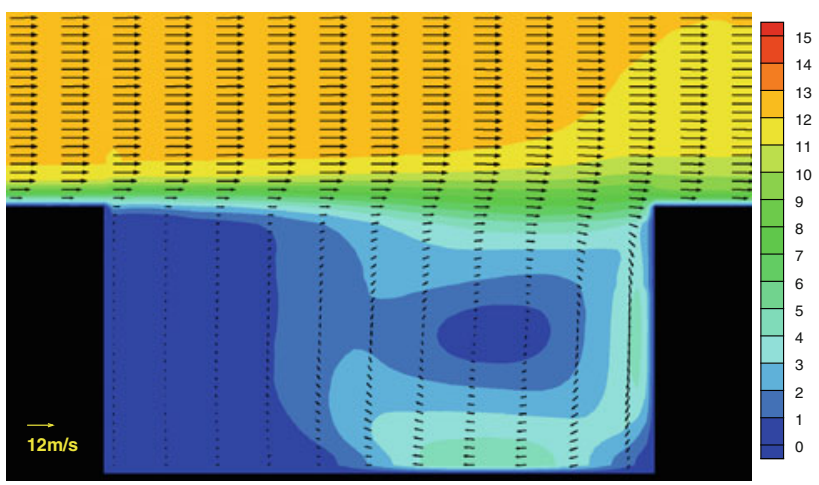

(a) $12 \mathrm{~m} / \mathrm{s}$

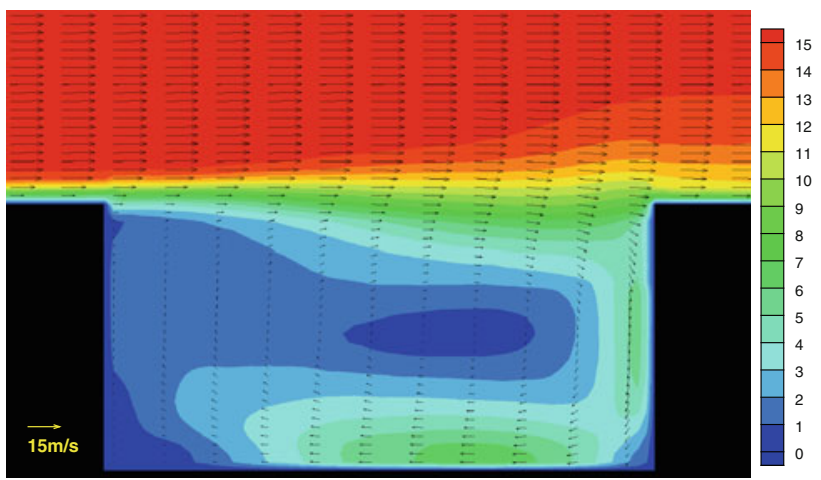

(b) $15 \mathrm{~m} / \mathrm{s}$

Fig. 7 Mean velocity contours and vectors $(\mathrm{m} / \mathrm{s})$. In the free-stream direction is shown one vector every six

region in the lower left corner; see Fig. 7. The recirculating flow inside the cavity impinges on the separating laminar shear layer, determines its destabilization and triggers the development of vortical structures that convect downstream and then impinging on the aft cavity wall as can be seen in the time series of Figs. 8 and 9. There are significant differences in the flow features for the two flow regimes we investigated. Figure 7 shows how at $15 \mathrm{~m} / \mathrm{s}$ the recirculation region inside the cavity is larger than at $12 \mathrm{~m} / \mathrm{s}$. This, together with the fact that the separating boundary layer is thinner at the higher velocity, makes the destabilization of the separated shear layer to occur earlier upstream and to develop in larger structures that will

Table 2 Settings of the interrogation domains in the various flow domains indicated in Fig. 5

\begin{tabular}{|c|c|c|c|c|}
\hline Zone & Pass 1 & Pass 2 & Pass 3 & Pass 4 \\
\hline 1. Free flow & $32 \times 32, \mathrm{ps}$ & $16 \times 16$ & $16 \times 16$ & none \\
\hline 2. Incoming boundary layer & $64 \times 8$ & $32 \times 4$ & $16 \times 4$ & $16 \times 4$ \\
\hline 3. Shear layer & $64 \times 64$, wd & $32 \times 32, w d$ & $16 \times 16, w d$ & $16 \times 16, \mathrm{wd}$ \\
\hline 4. After the cavity & $64 \times 32, w d$ & $32 \times 16, w d$ & $16 \times 16, w d$ & $16 \times 16, w d$ \\
\hline 5. Inside the cavity & $32 \times 32$, wd & $16 \times 16$, wd & $16 \times 16, \mathrm{wd}$ & none \\
\hline
\end{tabular}

Everywhere was used a $50 \%$ overlap of the interrogation windows. $p s$ preshift, $w d$ window deformation 


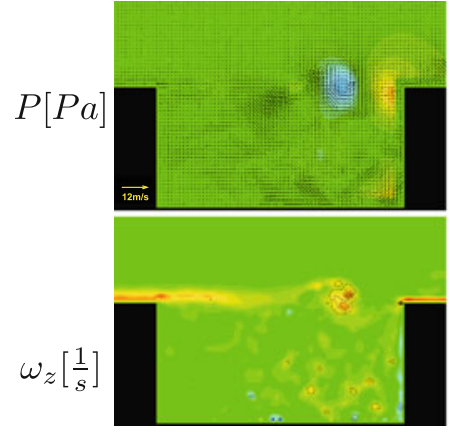

$t_{1}$
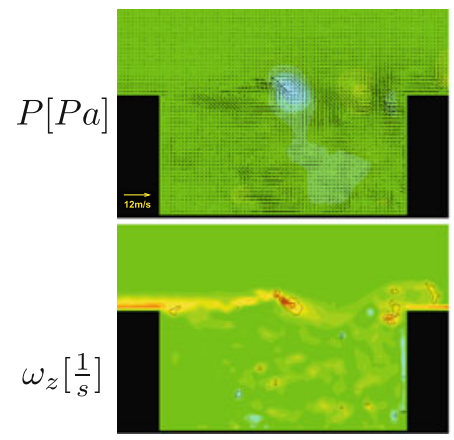

$t_{5}$

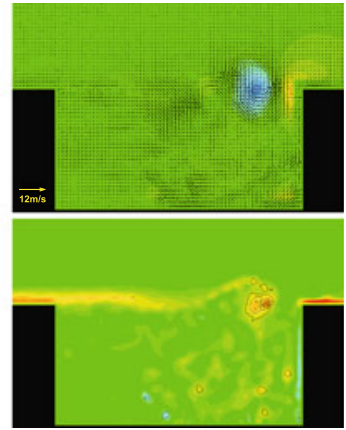

$t_{2}$
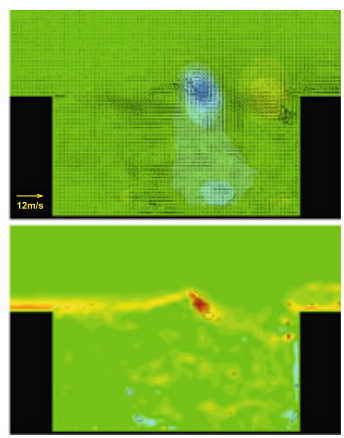

$t_{6}$
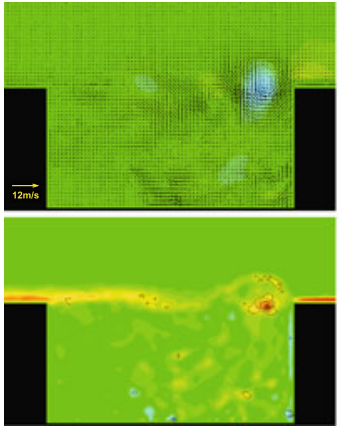

$t_{3}$
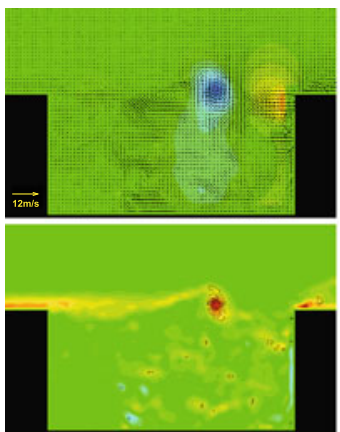

$t_{7}$
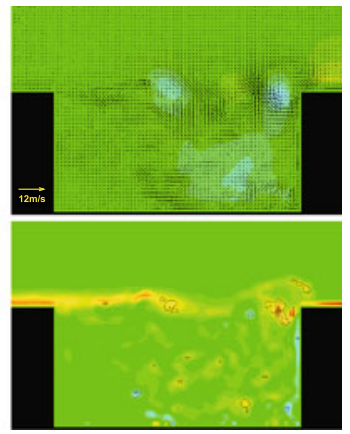

$t_{4}$
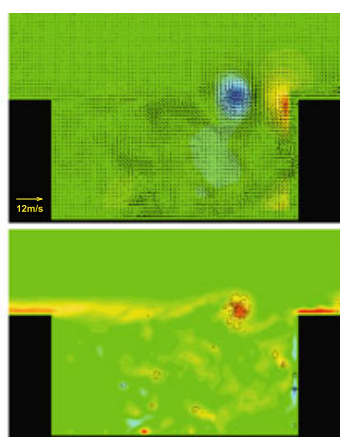

$t_{8}$

Fig. $812 \mathrm{~m} / \mathrm{s}$ : Instantaneous pressure and fluctuating velocity. Corresponding out of plane vorticity and $\lambda_{c i}$. $\Delta t=320 \mu \mathrm{s}$

impinge on the cavity wall. Those will lead to an higher amplitude of the pressure fluctuations and therefore to higher acoustic noise levels.

\section{Pressure from PIV data}

In Figs. 8 and 9 are shown the instantaneous fluctuating pressure distributions computed from the PIV data. The snapshots also show the out-plane vorticity component at the same instant in time (Figs. 10, 11). The vortical structures identified with the $\lambda_{c i}^{2}$ criterion, as well as by instantaneous fluctuating velocity vector field, (Jeong and Hussain 1995), correspond to low-pressure areas (indicated by the blue contours). Figure 12 shows the root mean square of the pressure. These plots are important, since they indicate the location from which most of the sound originates. In the surface integral of Eq. 4 there are two source terms: the pressure itself, with an intensity decaying

\footnotetext{
$2 \lambda_{c i}$, or swirling strength, is related to the eigenvalues of the velocity gradient tensor. In practice, a high level of the swirling strength indicates a location around which the streamlines are closed, which is typically the location of a vortex core.
}

in proportion to the square of the distance, and its time derivative, with an intensity decaying linearly with the distance. The sound that propagates further away from the source is then due to the pressure fluctuations at the walls. Figure 12 indicates that the pressure fluctuations at the aft wall of the cavity are larger, especially in the stagnation region. Figures 13 and 14 display the power spectra for the pressure fluctuations from different microphones positioned at the cavity walls and of the pressure fluctuation computed from the PIV data at the nearest location available. All the pressure power spectra shown in this paper are the averages of the power spectra of segments of the original time signals. Each time signal was divided in 8 segments of equal length with a 50\% overlap. Each segment is windowed with a Hamming window of the same length as the segment. The power spectra of the individual segment is then computed through the Fast Fourier Transform algorithm and then the power spectra of all segments are averaged to reduce the variance in the estimated power spectrum. For the position of the microphones refer to Fig. 4.

The computation of the pressure from the PIV data close to the cavity walls is a difficult task. First of all, the quality of the particle images deteriorates near the walls because of 


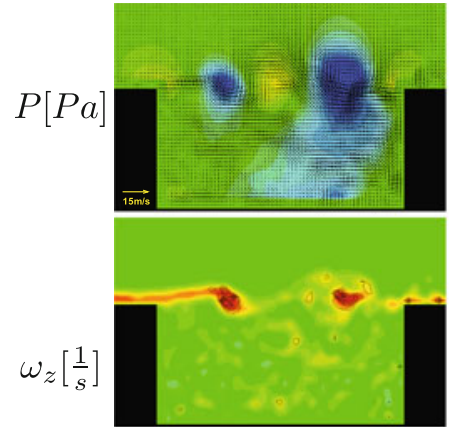

$t_{1}$
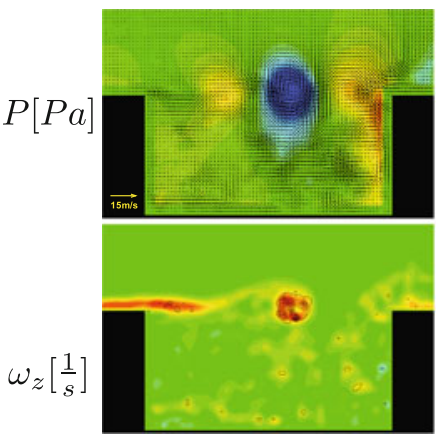

$t_{5}$

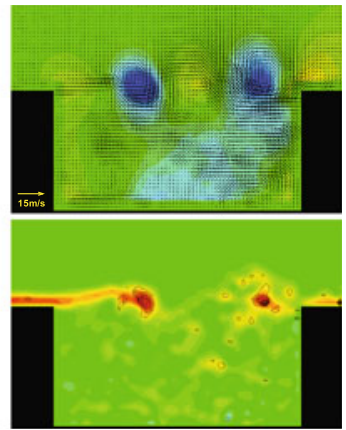

$t_{2}$
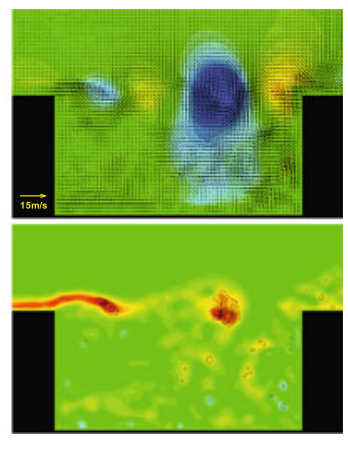

$t_{6}$

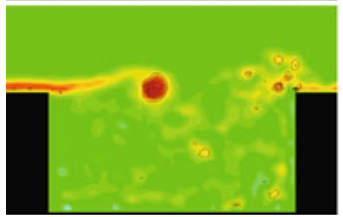

$t_{3}$
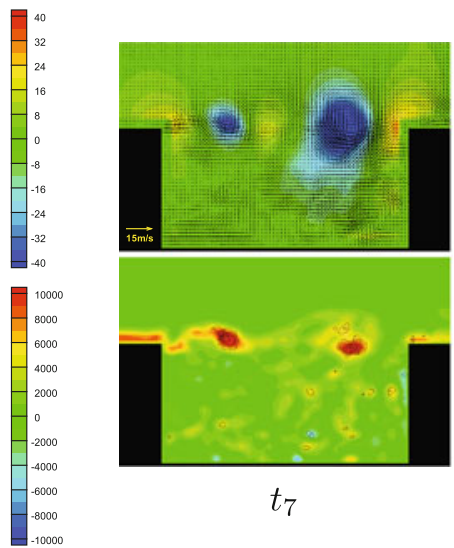

$t_{7}$
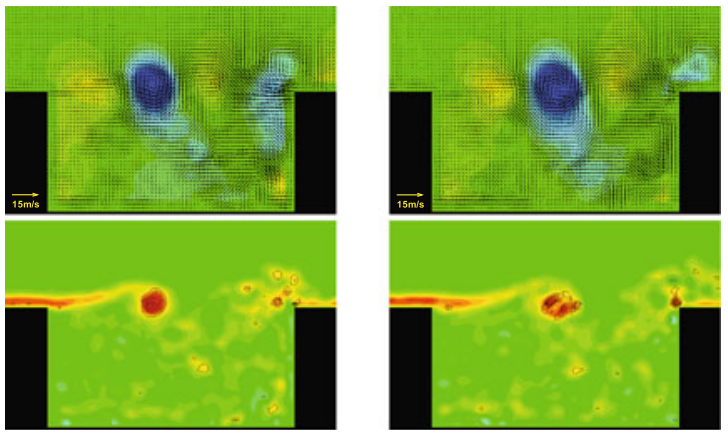

$t_{4}$

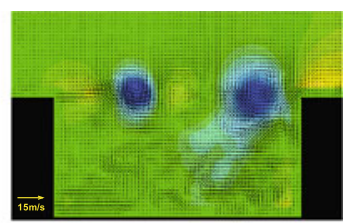

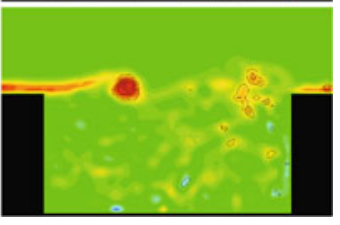

$t_{8}$

Fig. $915 \mathrm{~m} / \mathrm{s}$ : Instantaneous pressure and fluctuating velocity. Corresponding out of plane vorticity and $\lambda_{c i}$. $\Delta t=320 \mu \mathrm{s}$

light reflections from the cavity surface. Second, velocities are small (ideally zero at the wall), so the relative uncertainty of the measurement becomes larger. Finally, very close to the cavity walls the viscous effects, which are presently neglected, may be important. Nevertheless, results show that in the region considered as the main sound source, i.e. where the shear layer impinges on the aft wall of the cavity, the computation of the hydrodynamic pressure from the PIV data gives an adequate estimate: the pressure measurements from the microphones and the pressure signal determined from PIV data show similar distributions. In both cases, the main tonal component and the first harmonic are present. The broad band signal though is quite different at all positions except at microphone No. 11, corresponding to the stagnation area where the shear layer impinges. We think this mismatch between the direct microphone measurement and the indirect computation from the PIV data is due to the lower signal to noise ratio of the data away from the stagnation region. The velocities at the bottom of the cavity are in fact quite low and the relative error, given the measurement error in the PIV data (typically about 0.1 pixels, (Westerweel 2000)), is high. It is possible to see in Figs. 13 and 14 that the PIV spectra are "flat" over the full frequency range and at comparable levels for microphones No. 4 and No. 9. That indicates that the values can be attributed to random noise (i.e. constant at all frequencies) and not to physical pressure values. On the other hand, at microphone No. 11, being in the stagnation region, the values are higher than the anticipated noise level, and the two spectra show an excellent agreement. The same reason probably explains the lower amplitude of the main peak in the PIV pressure signal with respect to the one from the microphone.

\section{Acoustic emission with Curle's analogy}

We compute the acoustic pressure field with Curle's analogy, in the formulation expressed in Eq. 4. The integration is done using the rectangle method with midpoint approximation. Since we have data only in the spanwise median plane of our model, the cavity is considered to radiate homogeneously across the span. We consider every source location in the computation, also from spots that are not in a direct view of the listener. In fact, every source parcel, radiating spherically, affects the whole sound field. The path followed by the propagating waves in the proximity of any solid boundaries, provided that the 


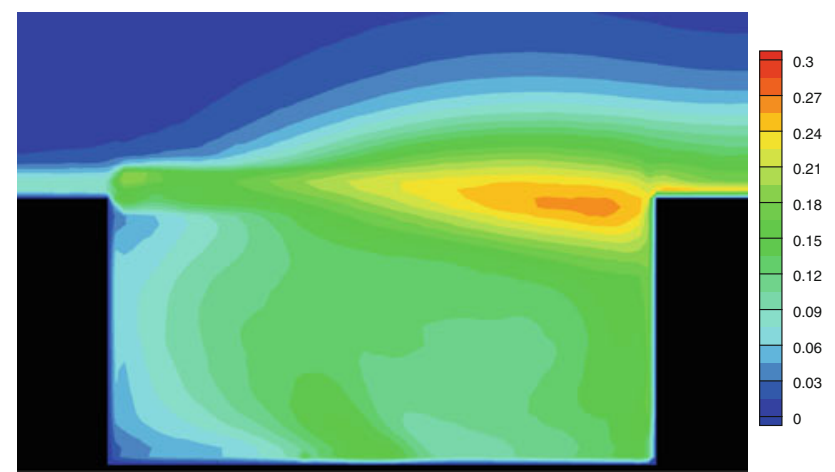

(a) $12 \mathrm{~m} / \mathrm{s}$

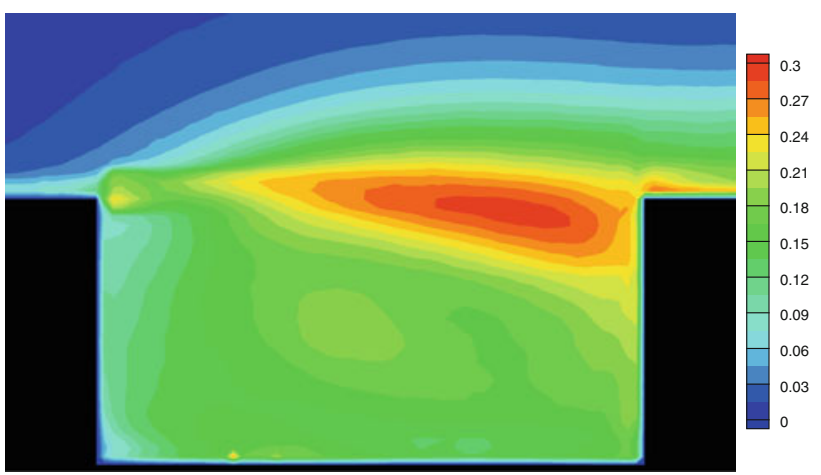

(b) $15 \mathrm{~m} / \mathrm{s}$

Fig. 10 Turbulent intensities normalized to the free stream velocities

solid body is compact, can be expressed as the one from a velocity potential flow, as described by Howe (2003). Since the cavity dimensions are much smaller than the typical wavelength ${ }^{3}$, the distance between the source parcel and the listener position along the real path can be well approximated by the linear distance, $r$. Particular attention must be put in the treatment of the boundary of the domain. The exact solution of Eq. 4 would involve integration over the entire semi-infinite plane before and after the cavity. But far away from the cavity the source intensities are weak and do not contribute significantly to the acoustic field. We have to take into account that our experimental field of view has a finite dimension. It extends over half a cavity length before and after the cavity. Thus, it includes most of the significant sources inside and near the cavity. Their intensities at the boundaries, though, are still quite high, and the jumps at the edges of the integration domain could generate spurious sources in the computation. We therefore apply a window to the computational domain to gradually reduce the intensity of the source terms approaching the boundaries. Figure 15 shows the power spectra of a microphone

\footnotetext{
$3 \lambda=\frac{a_{0}}{f}$, being $\lambda$ the typical wavelength, $a_{0}$ the speed of sound and $f$ the main frequency tone. So $\lambda=0.75 \mathrm{~m}$ for the $12 \mathrm{~m} / \mathrm{s}$ free-stream velocity flow and $\lambda=0.68 \mathrm{~m}$ for the $15 \mathrm{~m} / \mathrm{s}$ one.
}

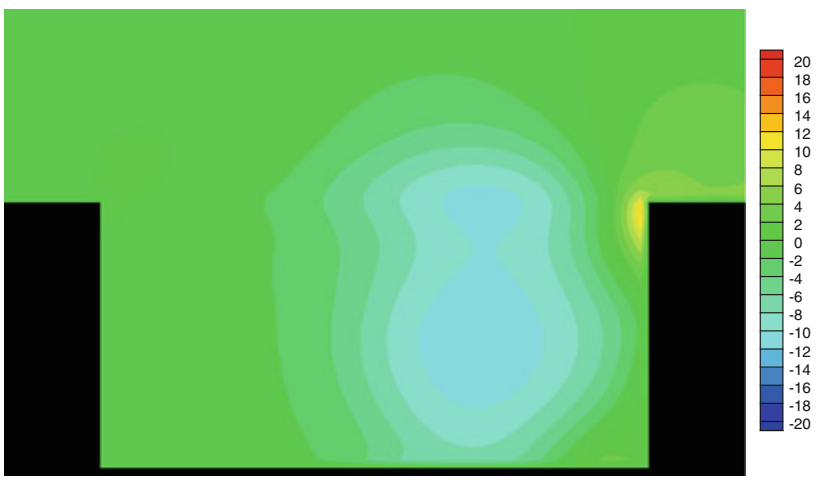

(a) $12 \mathrm{~m} / \mathrm{s}$

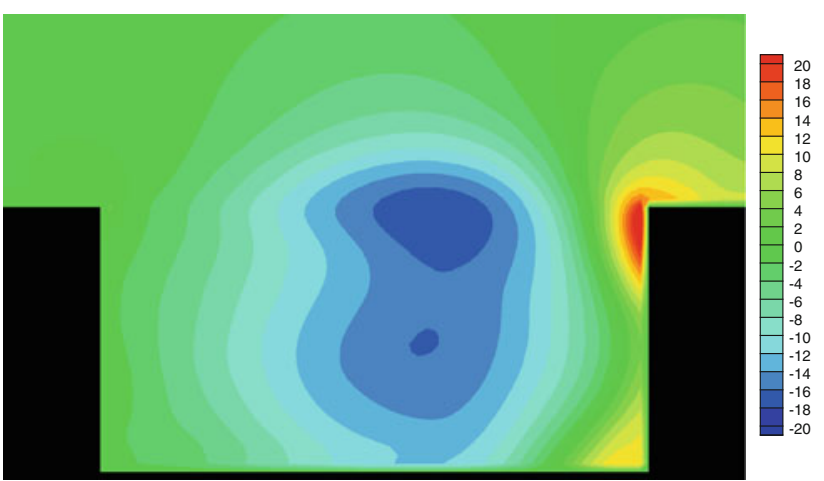

(b) $15 \mathrm{~m} / \mathrm{s}$

Fig. 11 Mean pressure contours (Pa)

positioned $0.5 \mathrm{~m}$ above the cavity (for the position refer to Figs. 16 and 17, position A) and of the acoustic signal computed with Curle's analogy at the same location. From these data we can see that the broadband signal is captured reasonably well. Both the main frequency peak and the first harmonic peak are detected. Their amplitude is lower compared to the microphone data, probably due to an over-estimate of the measured sound rather than to an under-estimate of the computation from the PIV data. The dimensions of the wind tunnel used in the experiments may in fact suggest the presence of standing waves amplifying the signal in that range of frequencies. The broadband signal is captured well at the low frequencies, but not at the higher ones where results appear to be higher than the microphone data. This result is confirmed by the measurements at different locations. This mismatch is probably due to two different reasons. Firstly, the PIV measurement have higher noise levels than the microphones measurements. This translates in an higher broadband power spectrum signal. Secondly, the difference in the results may be due to the hypotheses we made in the computation of the sound field. We considered the cavity as radiating homogeneously along the whole span, while we only have measurements in a single plane. This is a reasonable approximation for the larger structures that 


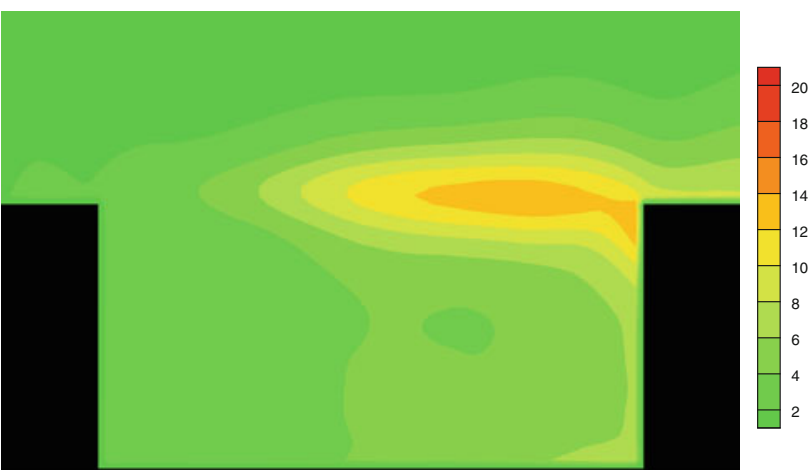

(a) $12 \mathrm{~m} / \mathrm{s}$

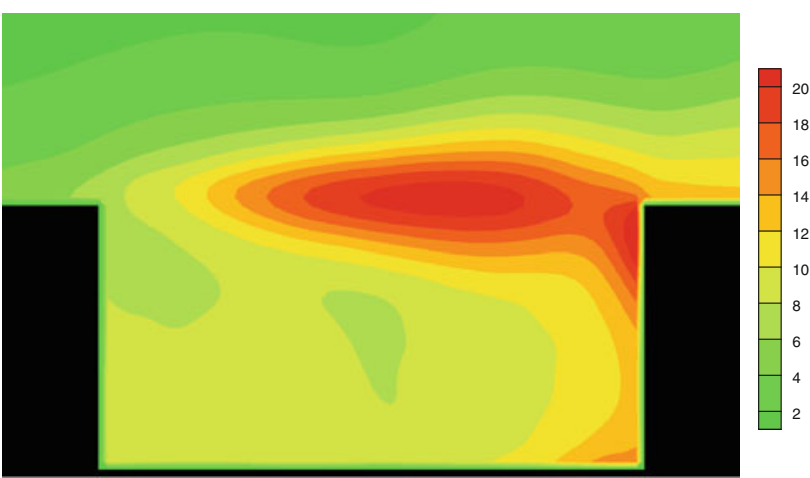

(b) $15 \mathrm{~m} / \mathrm{s}$

Fig. 12 Root mean square pressure contours $(\mathrm{Pa})$

are responsible for the tonal component of the spectrum, but it is less valid for the smaller structures still present in our flow. Considering also the small structures as homogeneous along the whole span generates therefore high frequency components in the computation of the sound emitted that in reality, where this coherence along the span does not exist, cancel each other out. Figure 17 shows the sound pressure level ${ }^{4}$ in the region above the cavity (the cavity is centered with respect to the horizontal axis and the position $y=0$ in the vertical direction corresponds to the lead of the cavity). The cavity seems to radiate as a dipole, peaking in the upstream and downstream directions with the level larger in the upstream direction at an angle of $120.5^{\circ}$ for the lower velocity and $126^{\circ}$ for the higher one. This results are consistent with what analytically described by Howe (2004) and with the results shown by Colonius et al. (1999) and Haigermoser (2009). Table 3 shows the sound pressure level for the two test velocities at two different locations above the cavity, where microphones were placed. The results that were obtained by the

\footnotetext{
${ }^{4} S P L_{d b}=20 \log _{10}\left(\frac{P_{R M S}}{P_{\text {ref }}}\right)$ where $P_{\text {ref }}=20 \mu \mathrm{Pa}$ is a reference value corresponding to the human audible threshold.
}

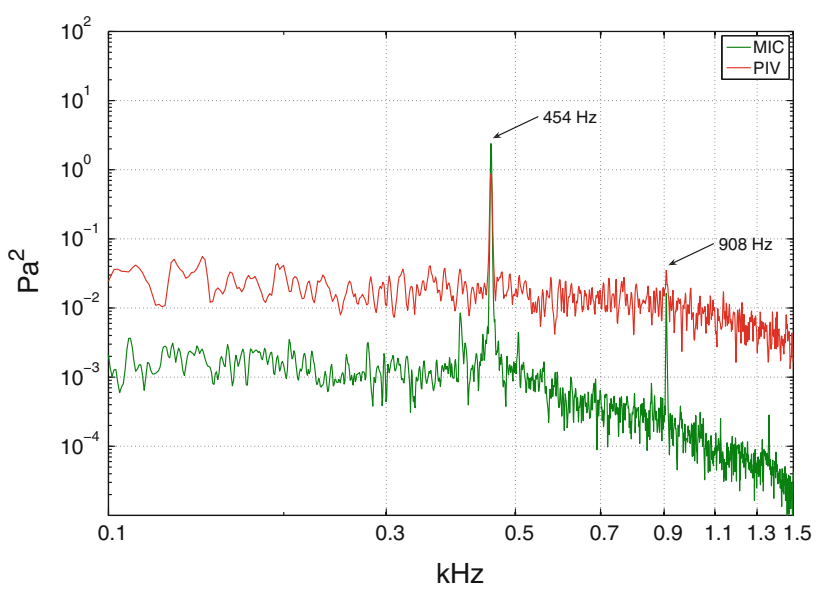

(a) Mic n.4

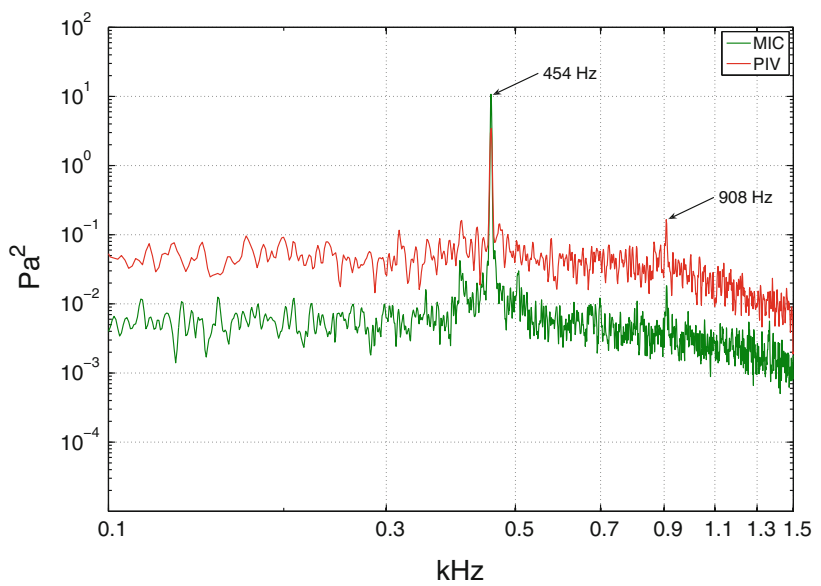

(b) Mic n.9

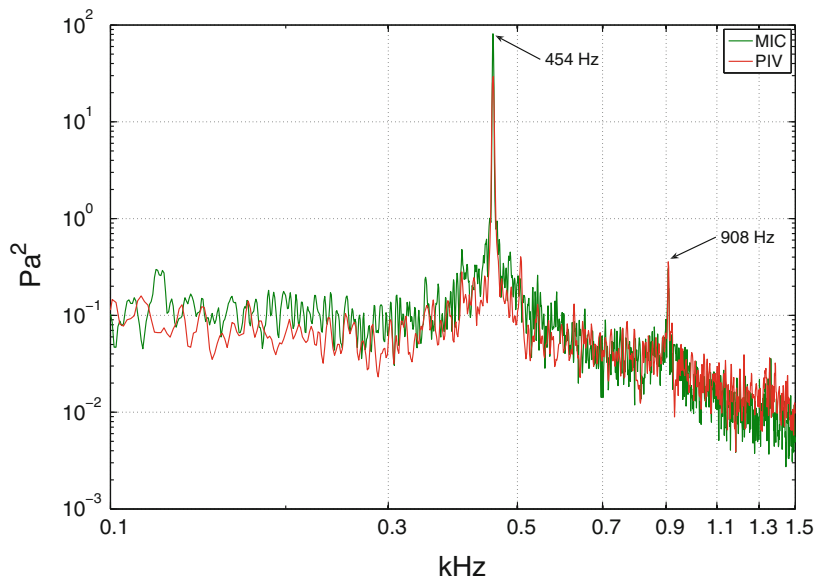

(c) Mic n.11

Fig. 13 Power spectra of pressure signal from microphones 4, 9 and 11 and of pressure computed from PIV at the same location at $12 \mathrm{~m} / \mathrm{s}$

application of Curle's analogy to the PIV data are within two to three dB lower than the direct measurement of the microphones. This can be regarded as a good approximation of the sound field. 


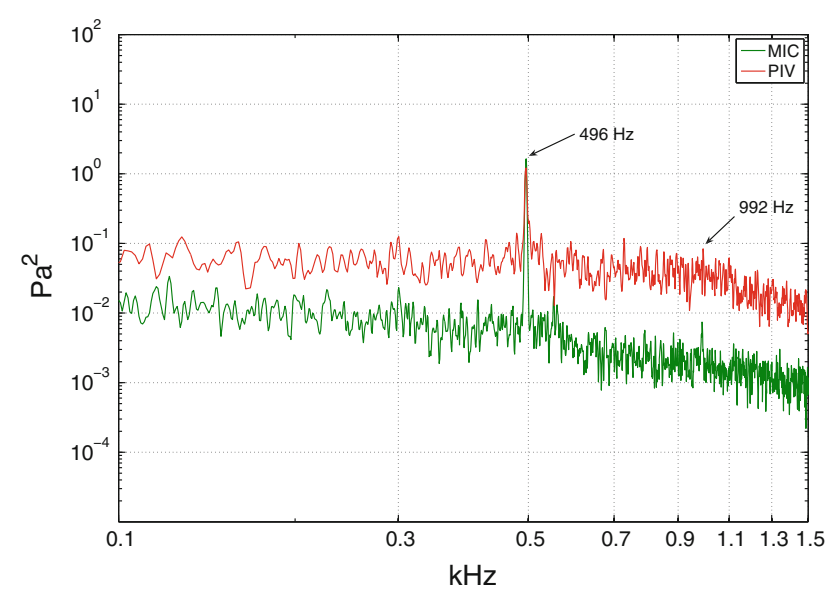

(a) Mic n.4

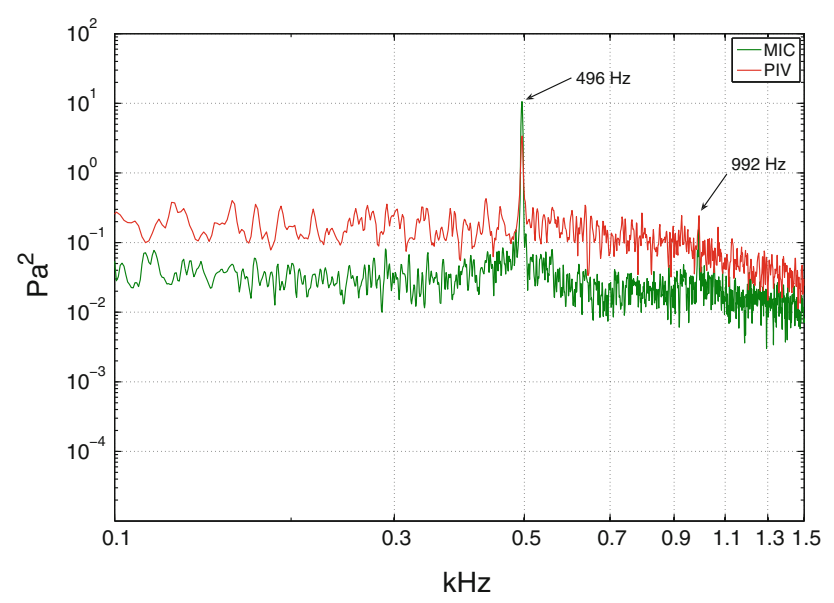

(b) Mic n.9

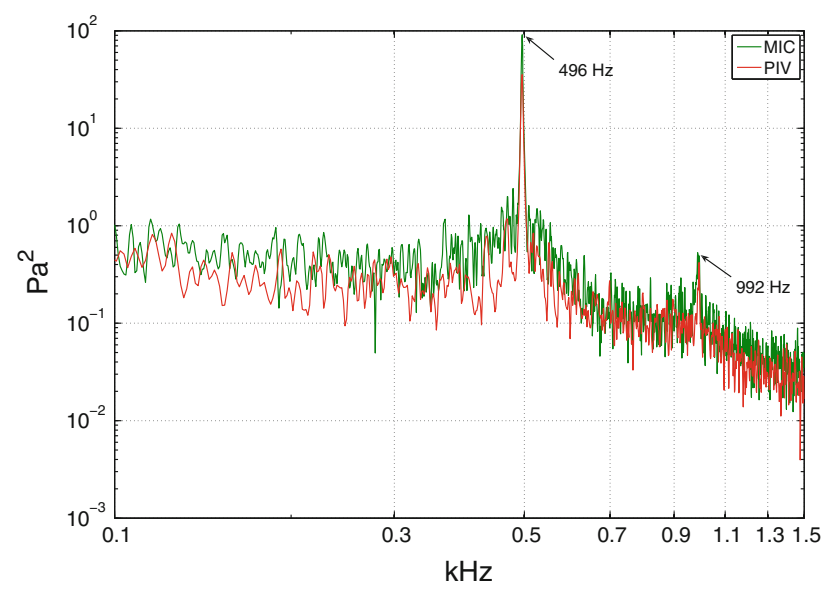

(c) Mic n.11

Fig. 14 Power spectra of pressure signal from microphones 4, 9 and 11 and of pressure computed from PIV at the same location at $15 \mathrm{~m} / \mathrm{s}$

\section{Spanwise coherence of the cavity flow}

As explained in Sect. 6, we assume that the flow is radiating coherently along its span in the computation of the

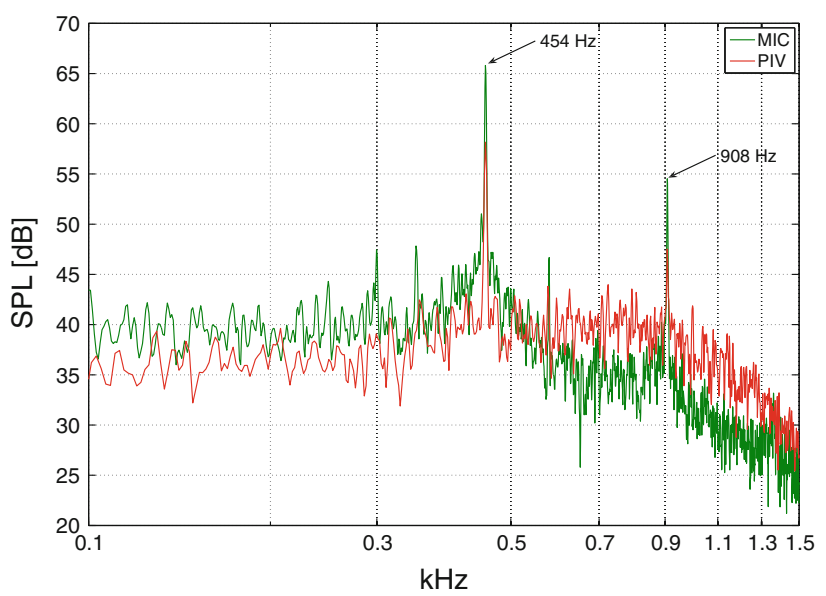

(a) $12 \mathrm{~m} / \mathrm{s}$

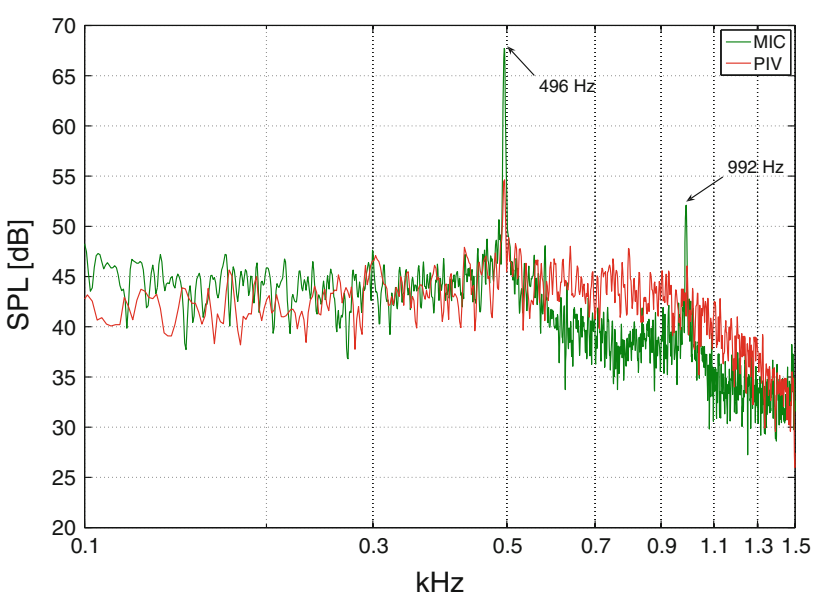

(b) $15 \mathrm{~m} / \mathrm{s}$

Fig. 15 Power spectra of sound signal from a microphone and of sound computed from PIV at the same location

sound field. This is a reasonable approximation for the larger structures that are responsible for the tonal component of the spectrum, but it is probably not valid for the smaller structures still present in our flow. We see the consequences of such an assumption in the spectra of Fig. 15, where the broadband signal is captured well at the low frequencies, but not at the higher ones. Here planar PIV-based results appear to be higher in amplitude than the microphone data. We take into consideration that the assumption made does not affect the results with respect to the purposes of this paper: i.e. the development and validation of a measurement technique. Nevertheless, we include here results showing the out-of-plane velocity component on a spanwise-streamwise plane positioned within the shear layer, just above the cavity lid (Fig. 18). These are the results from a stereoscopic measurement performed with two 4 megapixel cameras (LaVision Imaging Pro $\mathrm{X}$ ), giving a higher resolution than the time resolved measurement. The setup is shown in Fig. 19. 


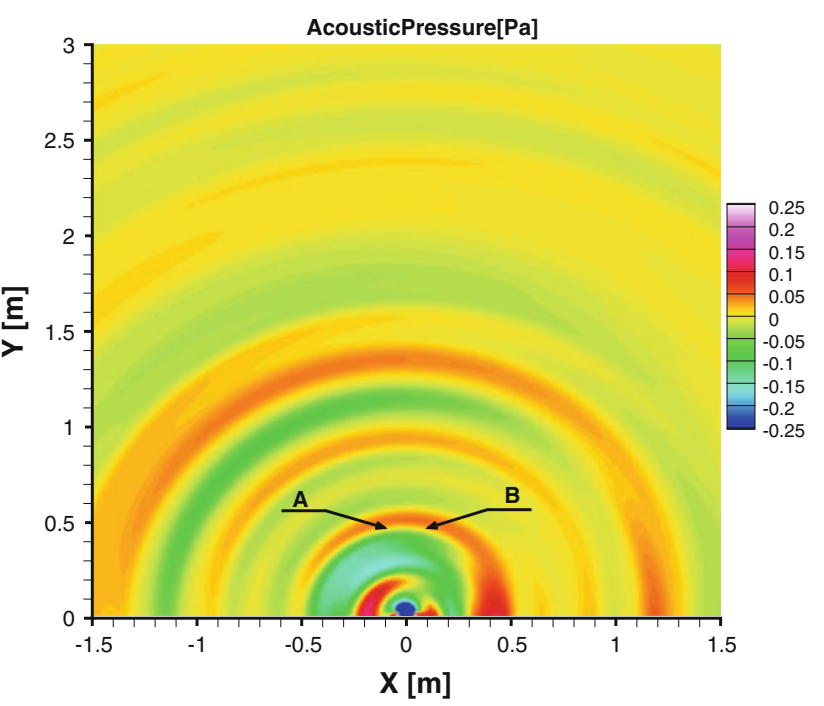

(a) $12 \mathrm{~m} / \mathrm{s}$

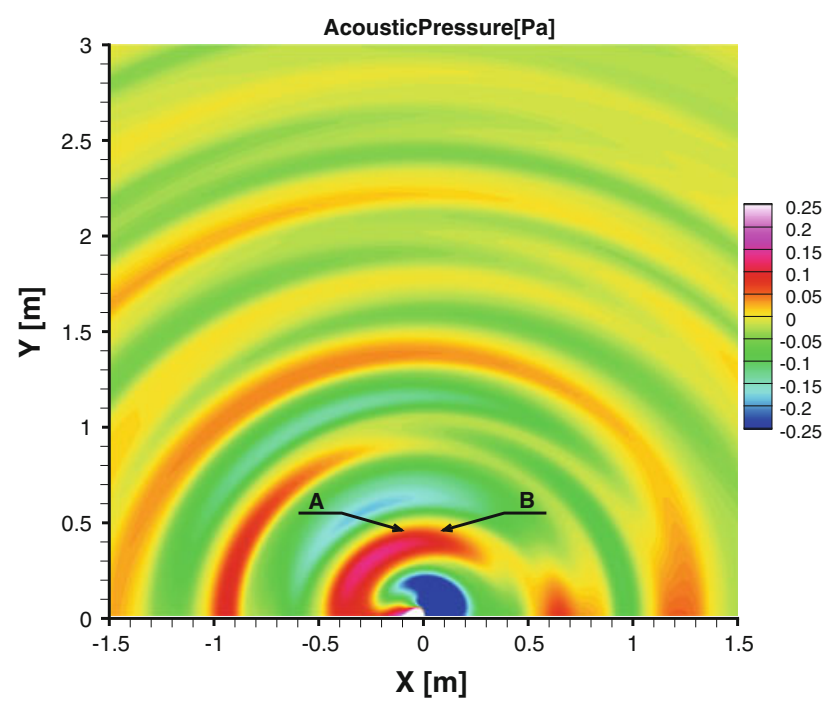

(b) $15 \mathrm{~m} / \mathrm{s}$

Fig. 16 Acoustic pressure field, $(\mathrm{Pa})$

From the measurements, little can be said about the smaller structures that are three dimensional and therefore not properly resolved by planar measurements. We can check to which extent the spanwise coherence assumption is valid for the large periodic structures. In Fig. 20 a typical structure can be clearly recognized as two parallel regions of strong positive and negative out-of-plane velocity. The two horizontal black lines represent the positions of the leading and trailing edges of the cavity. By detecting regions of high positive and negative out-of-plane velocity, we obtain a distribution of the positions of the periodic structures. Figure 21 shows the probability density function of the deviation of the structures from a pure two-dimensional vortex. Data are normalized with respect to the cavity length, L. The assumption of spanwise coherence, at

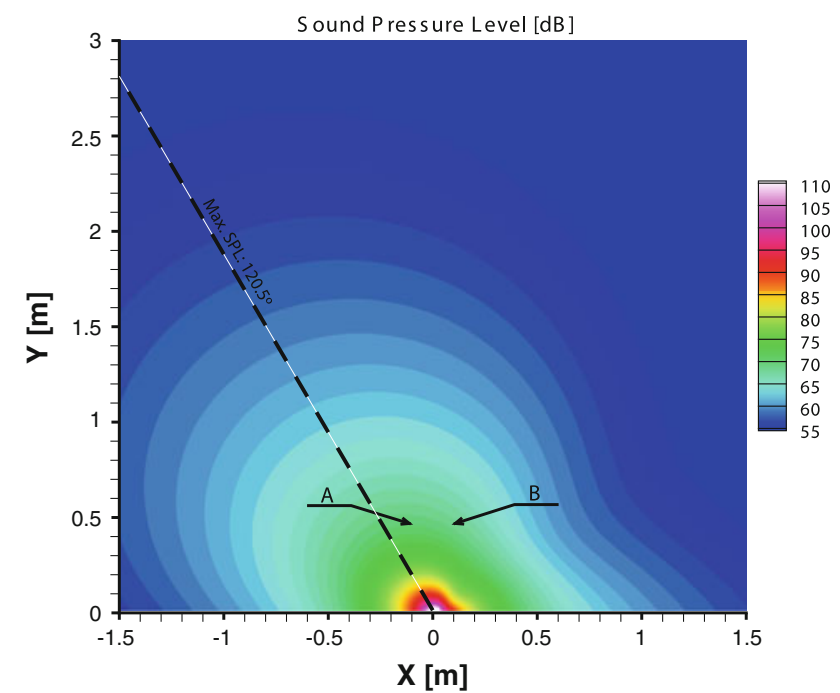

(a) $12 \mathrm{~m} / \mathrm{s}$

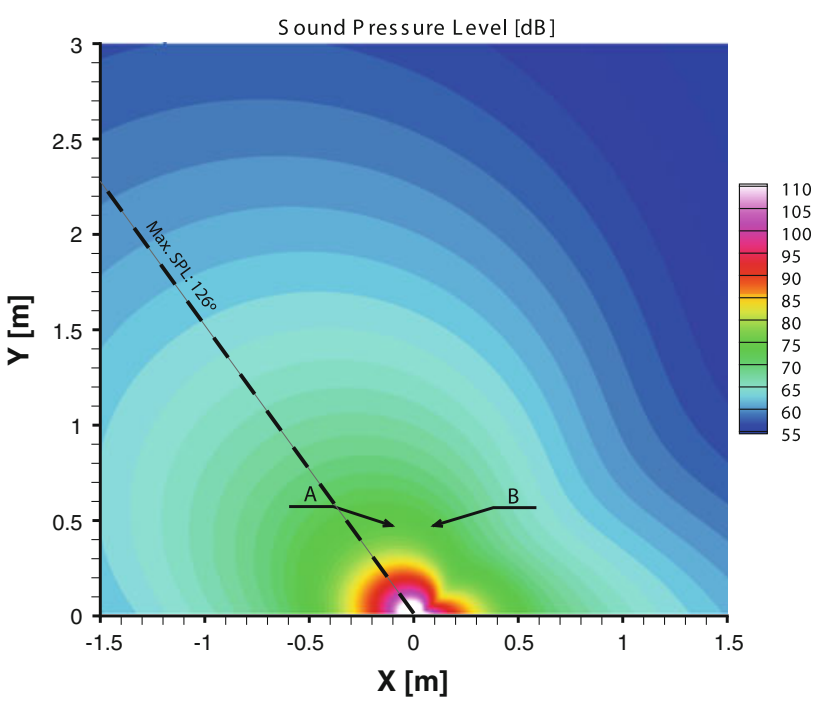

(b) $15 \mathrm{~m} / \mathrm{s}$

Fig. 17 Computed overall sound pressure level, (dB)

Table 3 Overall sound pressure levels

\begin{tabular}{llllll}
\hline$U_{0}(\mathrm{~m} / \mathrm{s})$ & \multicolumn{2}{l}{ Position $\mathrm{A}$} & & \multicolumn{2}{l}{ Position B } \\
\cline { 2 - 3 } \cline { 6 - 6 } & Mic $(\mathrm{dB})$ & PIV $(\mathrm{dB})$ & & Mic $(\mathrm{dB})$ & PIV $(\mathrm{dB})$ \\
\hline 12 & 73.23 & 70.65 & & 72.14 & 68.85 \\
15 & 78.87 & 74.98 & & 76.20 & 73.11 \\
\hline
\end{tabular}

least for the larger structures, seems to be reasonably satisfied since the $95 \%$ of samples deviate no more than 0.078 cavity lengths from an ideal spanwise coherent vortex.

\section{Conclusions}

In this work, we compute the pressure field from PIV data, and we apply Curle's analogy to compute the sound 


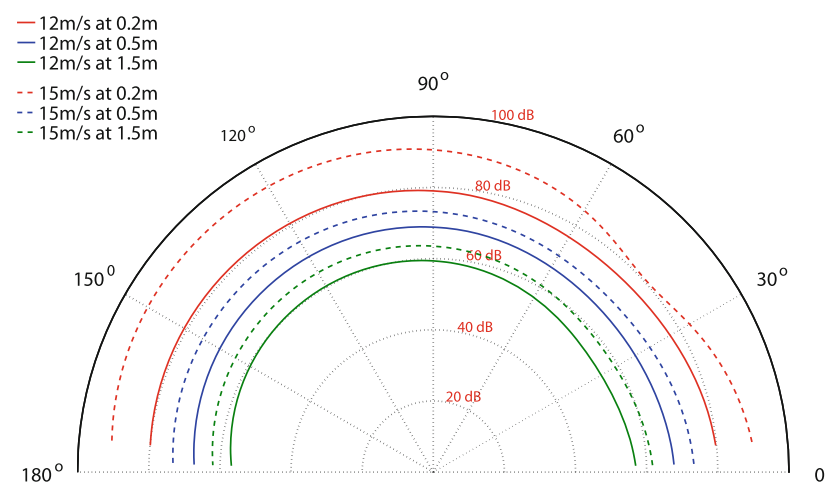

Fig. 18 Directivity chart for the overall sound pressure level. Continuous lines $12 \mathrm{~m} / \mathrm{s}$, Dashed lines $15 \mathrm{~m} / \mathrm{s}$

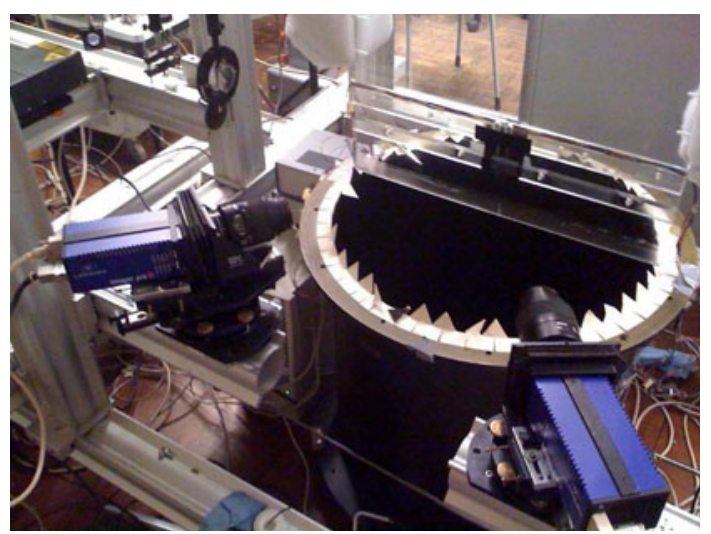

Fig. 19 Stereoscopic setup

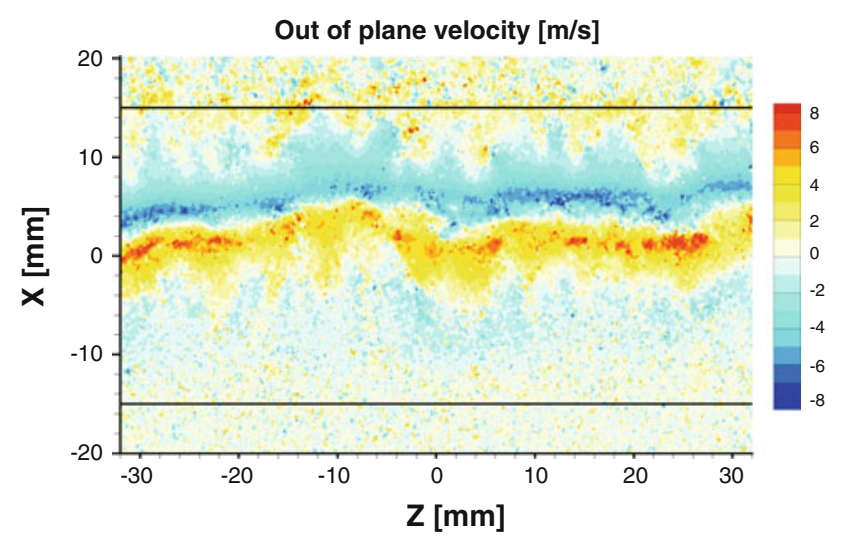

Fig. $20 U_{0}=12 \mathrm{~m} / \mathrm{s}$. Out-of-plane velocity component

emitted from a flow passing over a cavity. The aim is to find a way to directly relate the flow behavior to the produced acoustic noise. This will help considerably to understand the sound production mechanisms and to investigate possible ways to reducing it. A similar approach was applied previously by Lorenzoni (2008) on a rod-airfoil configuration. This gave promising result for this simple case. Recently Haigermoser (2009) tried the same approach on a geometry similar to ours, although the

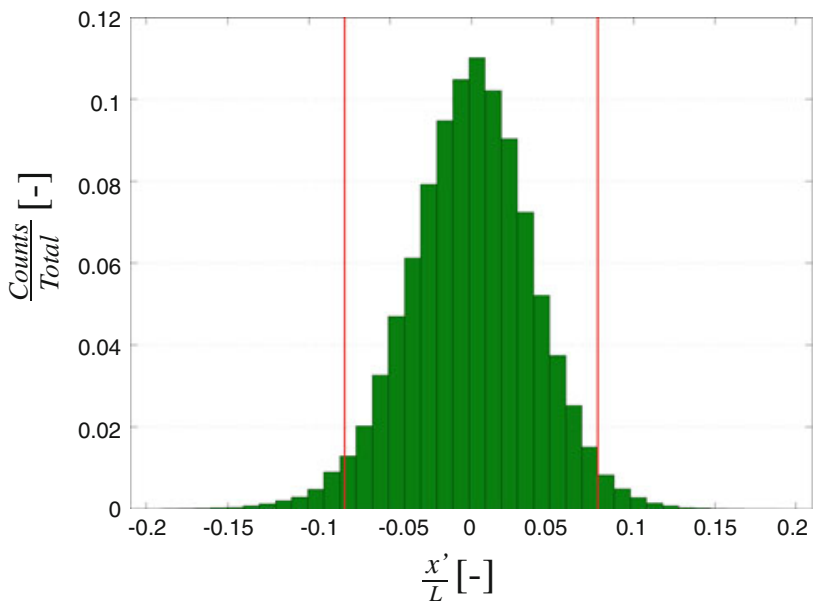

Fig. $21 U_{0}=12 \mathrm{~m} / \mathrm{s}$. PDF of deviation from mean vortex position normalized with the cavity length. The two vertical red lines represent the $2 \sigma$ threshold

measurement was carried out in water and without additional experimental validation using direct pressure measurements. Both the process of pressure calculation from PIV data and the application of Curle's analogy are affected by several uncertainties, and simplifying hypotheses have to be made in the process. For this reason, we measured the pressure fluctuations at the walls of the cavity and the sound emitted. We compared those values with the estimated quantities from the PIV data in order to validate the results and to check the range of validity of this approach. We find that the hydrodynamic pressure computed from the PIV data has a frequency spectrum that is comparable to that of the direct pressure measure at the cavity walls. The main frequency tone and the first harmonic are captured, although the amplitude was significantly underestimated. At the locations where the pressure fluctuation are very weak the noise level from the PIV measurements becomes significant and affects the reliability of the sound computation. We then solve Curle's analogy using the pressure and its time derivative at the walls as source terms. The power spectra of the sound signal from the PIV data and from a microphone at the same location have a similar frequency distribution, but the amplitude of the main tone is lower while the signal at high frequencies is higher than in the microphone spectra. The mismatch in the amplitude of the tonal component is probably due to an over-estimation of the measured sound rather than to an under-estimate of the computation from the PIV data. The dimensions of the wind tunnel used in the experiments may suggest the presence of standing waves amplifying the signal in that range of frequencies. Further investigation is planned to validate this hypothesis. Other explanations for the mismatch could be the limited field-of-view for the PIV measurements, which may 
exclude important sources of sound and a propagation of the same problem seen in the hydrodynamic pressure spectra, even if in a much weaker way. The higher signal at the high frequencies is due to the higher noise of the PIV data compared to the noise level in the microphones and to the error arising from the assumption that the flow radiates coherently along the whole span of the cavity. In Sect. 7, we demonstrate that this hypothesis is valid for the larger flow structures, responsible for most of the sound, but that may not be true for the smaller flow structures. The overall sound pressure level falls in within $2-3 \mathrm{~dB}$ from the one measured with the microphones. The frequency at which the shear layer shedding occurs matches with results obtained by others, (Ahuja and Mendoza 1995; Block 1976; Gharib and Roshko 1987; Howe 1997, 2004; Larsson et al. 2004; Rowley et al. 2002; Suponitsky et al. 2005). As expected for this Mach number regime, (Curle 1955), the dipolar source of sound is strongly dominant. Therefore, the tonal component of the acoustic emission occurs at the same frequency as the shear layer hedding. Directivity of the emission matches with what analytically derived by Howe (2004) and with the results of previous studies of Colonius et al. (1999) and Haigermoser (2009). This work shows that the technique provides reasonable results and is promising especially for capturing the tonal components in the sound emission. Further work must be done in the computation of the pressure near the walls. An evaluation of the three-dimensional components of the flow should be made, and more advanced experimental approaches that allow their measurement, such as stereoscopic PIV or multi-plane PIV, can be considered.

Acknowledgments This work is part of the research programme of the Foundation for Fundamental Research on Matter (FOM), which is financially supported by the Netherlands Organization for Scientific Research (NWO).

Open Access This article is distributed under the terms of the Creative Commons Attribution Noncommercial License which permits any noncommercial use, distribution, and reproduction in any medium, provided the original author(s) and source are credited.

\section{References}

Ahuja KK, Mendoza J (1995) Effects of cavity dimensions, boundary layer, and temperature on cavity noise with emphasis on benchmark data to validate computational aeroacoustic codes. NASA report CR-4654

Block PJW (1976) Noise response of cavities of varying dimensions at subsonic speeds. NASA TN D-8351

Colonius T, Basu AJ, Rowley W (1999) Numerical investigation of the flow past a cavity. AIAA/CEAS Aeroacoustics Conference and Exhibit
Curle N (1955) The influence of solid boundaries upon aerodynamic sound. Proc R Soc Lond A 231: 505-514

De Kat R, Oudheusden van BW, Scarano F (2008) Instantaneous planar pressure field determination around a square-section cylinder based on time-resolved stereo-piv. 14th Int Symp on Applications of Laser Techniques to Fluid Mechanics Lisbon, Portugal, 07-10 July, 2008

Ffowcs JE, Hawkings DL (1969) Sound generation by turbulence and surfaces in arbitrary motion. Proc R Soc Lond A 264: 321-342

Gharib M, Roshko A (1987) The effect of flow oscillations on cavity drag. J Fluid Mech 177: 501-530

Grace SM, Dewar WG, Wroblewski DE (2004) Experimental investigation of the flow characteristics within a shallow wall cavity for both laminar and turbulent upstream boundary layers. Exp Fluids 36: 791-804

Haigermoser C (2009) Application of an acoustic analogy to piv data from rectangular cavity flows. Exp Fluids 47: 145-157

Haigermoser C, Vesely L, Novara M, Onorato M (2008) A timeresolved particle image velocimetry investigation of a cavity flow with a thick incoming boundary layer. Phys Fluids 20

Howe MS (1997) Low strouhal number instabilities of flow over apertures and wall cavities. J Acoust Soc Am 102: 772-780

Howe MS (2004) Mechanism of sound generation by low mach number flow over a wall cavity. J Sound Vib 273: 103-123

Howe MS (2003) Theory of vortex sound. Cambridge University Press, Cambridge

Jeong J, Hussain F (1995) On the identification of a vortex. JFM 285: 69-94

Larchevêque L, Sagaut P, Labbé O (2007) Large-eddy simulation of a subsonic cavity flow including asymmetric three-dimensional effects. J Fluid Mech 577: 105-126

Larsson J, Davidson L, Olsson M, Eriksson L (2004) Aeroacoustic investigation of an open cavity at low mach number. AIAA J 42(12)

Lighthill MJ (1952) On sound generated aerodynamically, part 1: General theory. Proc R Soc Lond A 211: 564-587

Liu X, Katz J (2006), Instantaneous pressure and material acceleration measurements using a four-exposure piv system. Exp Fluids 41: 227-240

Lorenzoni V (2008) Aeroacoustic investigation of rod-airfoil noise based on time-resolved piv. Master of Science Thesis-Faculty of Aerospace Engineering, Delft University of Technology

Moore P, Lorenzoni V, Scarano F (2009), Comparison of two techniques for aeroacoustic determination from a rod-airfoil piv experiment. 8th International Symposium on Particle Image Velocimetry-PIV09

Rossiter JE (1964) Wind tunnel experiments on the flow over rectangular cavities at subsonic and transonic speeds. ARC Reports and Memoranda 3438

Rowley W, Colonius T, Basu AJ (2002) On self-sustained oscillations in two-dimensional compressible flow over rectangular cavities. J Fluid Mech 455: 315-346

Suponitsky V, Avital E, Gaster M (2005) On three-dimensionality and control of incompressible cavity flow. Phys Fluids 17

Tam CKW, Block PJW (1978) On the tones and pressure oscillations induced by flow over rectangular cavities. J Fluid Mech 89: 373-399

Westerweel J (2000) Theoretical analysis of the measurement precision in particle image velocimetry. Exp Fluids 29: S3-S12

Westerweel J, Dabiri D, Gharib M (1997) The effect of a discrete window offset on the accuracy of cross-correlation analysis of piv recordings. Exp Fluids 23: 20-28 